\title{
Cognition according to Quantum information: Three Epistemological Puzzles Solved
}

\author{
Vasil Penchev, vasildinev@gmail.com \\ Bulgarian Academy of Sciences: Institute of Philosophy and Sociology: \\ Dept. of Logical Systems and Models
}

\section{Abstract:}

The cognition of quantum processes raises a series of questions about ordering and information connecting the states of one and the same system before and after measurement: Quantum measurement, quantum invariance and the nonlocality of quantum information are considered in the paper from an epistemological viewpoint.

The adequate generalization of 'measurement' is discussed to involve the discrepancy, due to the fundamental Planck constant, between any quantum coherent state and its statistical representation as a statistical ensemble after measurement. Quantum invariance designates the relation of any quantum coherent state to the corresponding statistical ensemble of measured results.

A set-theory corollary is the curious invariance to the axiom of choice: Any coherent state excludes any well-ordering and thus excludes also the axiom of choice. However the above equivalence requires it to be equated to a well-ordered set after measurement and thus requires the axiom of choice for it to be able to be obtained.

Quantum invariance underlies quantum information and reveals it as the relation of an unordered quantum "much" (i.e. a coherent state) and a well-ordered "many" of the measured results (i.e. a statistical ensemble). It opens up to a new horizon, in which all physical processes and phenomena can be interpreted as quantum computations realizing relevant operations and algorithms on quantum information. All phenomena of entanglement can be described in terms of the so defined quantum information. Quantum invariance elucidates the link between general relativity and quantum mechanics and thus, the problem of quantum gravity.

The nonlocality of quantum information unifies the exact position of any space-time point of a smooth trajectory and the common possibility of all space-time points due to a quantum leap. This is deduced from quantum invariance.

Epistemology involves the relation of ordering and thus a generalized kind of information, quantum one, to explain the special features of the cognition in quantum mechanics.

Key words: axiom of choice, entanglement, quantum information, quantum invariance, qubit 
Quantum mechanics offers an abundance of epistemological surprises seeming paradoxical at first glance, but contradicting the prejudices only. Three of them, linked to quantum measurement, quantum invariance, and the nonlocality of quantum information, will be subject of this paper. They refer to the relation of ordering before and after measurement, which is way of cognition of any quantum system, as well as the deducible conclusions and interpretations restricted to epistemology.

\section{Quantum Measurement}

The concept of measurement in quantum mechanics is seen from a few philosophical and mathematical viewpoints further: quantum invariance, the foundation of mathematics and the axiom of choice, and quantum measure.

\subsection{Quantum measurement and the axiom of choice:}

The theorem of Kochen - Specker (1968) and yet John von Neumann's (1932) one before it require the absence of any hidden variables in any quantum state before measurement and thus they exclude any well-ordering in it. In fact any well-ordering before measurement is excluded by any theory utilizing differential equations to describe the states in the studied area. It can happen only if the axiom of choice is added externally for the smooth continuum necessary for any differential equation cannot be well-ordered otherwise than by it. However the difference between a point of the continuum and a corresponding point in a well-ordered subset of it can converge to zero. That way out is forbidden to quantum mechanics because of the Plank constant for it imposes a finite difference between the states before and after measurement. Furthermore any set of measured results is well-ordered, e.g. by the exact time of recording in a central computer.

If one combines the Kochen - Specker theorem with the well-ordering after measurement, the well-ordering theorem equivalent to the axiom of choice is unavoidable: So quantum measurement involves necessarily the axiom of choice. One can object that the sets before and after measurement are different so that the well-ordering theorem is irrelevant. In fact even then the mapping between a set, which cannot be well-ordered in principle, and that, which is always well-ordered, requires the axiom of choice for the Cartesian product between them to exist, of which the mapping is a subset. So the measurement of any quantum system or state designated shortly as 'quantum measurement' cannot be free of the axiom of choice.

Since the Kochen - Specker theorem does not admit the axiom of choice in any quantum system or state by itself, i.e. before measurement, and quantum measurement requires it as above, quantum mechanics has to be invariant to the axiom of choice: Any statement or equation in it has to be equally valid both if the axiom of choice is accepted or not.

\subsection{Quantum measurement and quantum invariance:}

This unique relation of quantum mechanics to the axiom of choice is due to the true fundament of it, wave-particle duality and thus, to involving quantum leaps in mechanical motion. One can speak of quantum invariance underlying the above noticed invariance to the axiom of choice for quantum measurement. The wave-particle duality has another side, which can be designated as the discrete-smooth duality of mechanical motion, since there is an exact correspondence of a wave function to a quantum leap (a discrete motion) as well as of a particle to a smooth trajectory (a world line). Thus that discrete-smooth duality is a kind of generalization as to Einstein's (1918) principle of relativity, which requires the invariance of all physical laws only to smooth relative motions (reference frames), so that it comprises already also quantum leaps.

That new and more general invariance is quantum invariance: It means that all physical laws have to be invariant to any, both discrete and continuous (smooth), transformation between 
two or more reference frames. The generalization pioneers the simplest pathway between general relativity and quantum mechanics, i.e. that of quantum gravity.

Any discrete motion does not allow defining a finite value of relative speed. If yet it is defined, this excludes to determine the distance of the leap. Both complementary restrictions constitute together the Heisenberg principle of uncertainty. This uncertainty is a new and unique physical variable, a free variable unboundable in principle, by a natural law such as uncertainty. Its physical dimension is action: The physical quantity of action is exceptional and singular since it is as the dimension of that unboundable free variable (in quantum mechanics) as the dimension of the corresponding bound variable (in general relativity) of the same name and dimension. Consequently what is conserved passing from each to the other theory is action in a rather extraordinary way of conservation: A dimensionless physical quantity like entropy is transformed in its reciprocal also dimensionless physical quantity like information and vice versa. Said philosophically, the disorder of entropy is transformed in the order of information and vice versa conserving action as in each of both theories as between them: The theory of quantum gravity turns out to be the theory of quantum information.

Furthermore the mutual transformation between entropy and information or between disorder and order in the framework of conservation of action describes well quantum measurement and quantum invariance, and even the invariance to the axiom of choice: Indeed quantum measurement transforms an initial fundamental disorder of coherent state into the order of a well-ordering of measured results, and quantum invariance means the equivalence of the disorder of probable values in a quantum leap and of the determined order of a smooth trajectory.

\subsection{Quantum measurement and the foundation of mathematics:}

The mutual transformation of order and disorder as the invariance to the axiom of choice deserves to be independently described for it can refer to the foundation of mathematics and thus to a new understanding how mathematics and physics are connected in reality and in quantum measurement. In fact the invariance to the axiom of choice is well known in the foundation of mathematics for a long time as the so-called paradox of Skolem (1922). He introduced the "relativity of the notion of set" meaning that any infinite set can be enumerated by the axiom of choice or even to be interpreted as a finite one. So a bridge exists from quantum measurement to a new, quantum foundation of mathematics. Its main idea is to borrow from nature the way how mathematics is founded in it generalizing the well-ordered set of natural numbers to that of qubits, which is equivalent to Hilbert space:

In other words, the idea is the founding set of natural numbers, which is always countable and thus it is noninvariant to the axiom of choice, to be replaced by the simplest one, which is invariant to the axiom of choice being uncountable and countable as a quantum coherent state before and after measurement. Such a one is Hilbert space, or the set of all well-ordered series of qubits. A point in it (or a sequence of qubits, or a wave function) can represent equally well both a coherent state before measurement and its corresponding statistical ensemble after measurement. That point is invariant to the transformation between entropy (E) and information (I) if and only if the definition of entropy and information is modified in a way to be invariant to the reciprocal transformation of their variables: $\mathrm{E}(x)=E\left(\frac{1}{x}\right)=I(x)=I\left(\frac{1}{x}\right)$. The Shannon type definition is not invariant so. However, the definition of physical quantity or observable in quantum mechanics by a selfadjoint operator is invariant just so. Consequently the latter is to be accepted as the relevant definition of information at least as to the quantum foundation of mathematics. 
That mathematics founded in this way cannot involve undecidable statements since decidability can be generalized as the invariance of entropy and information to the reciprocal transformation of their variables as above. Furthermore any statement Gödel codable can be coded as a series of qubits and thus undecidable statements cannot exist in that mathematics. Furthermore it cannot be divided from physics in the bridge of quantum mechanics and thus from reality. The conception of quantum measurement serves as the base of total decidability. It leads to some kind of quantum Pythagoreanism.

\subsection{Quantum measurement and quantum measure:}

The unit of quantum measurement is specific, quite different from the classical one even as the mathematical notion of measure: It is not a Lebesgue or Borel measure. This is quantum measure, which is complete as the former and universal as the latter. It is neither arbitrarily dimensional nor one-dimensional. It is three-dimensional. Its universal unit is a qubit isomorphic to a unit ball. This is the unit of quantum information: So quantum measure serves as the quantity of quantum information of any measured. Indeed it is really universal. It can measure equally well both the disorder of entropy and the order of information, both anything, which exists being actual, and nothing, which does not exist, being virtual, only possible and probable. Consequently, quantum measure can describe the "genesis from nothing" as a process, of course not as a process in time, because that is the genesis of time: For example, the "Big Bang" is not more than a visualization of quantum measure onto the "screen" of the usual understanding of measure.

Quantum measure is the covariant or complementary, or dual counterpart of quantum measurement in a sense: The change of quantum measure can be represented in the dual Hilbert space of the other Hilbert space of the change of the quantum-measured, which is conjugate, also entropy and information.

Historically, the quantum measure has arisen from the necessity of a common measure of discrete or continuous (smooth) motion in quantum mechanics. The classical decision supposes as the fundament to serve the latter measure, and the starting and final point of a quantum leap to be situated as points on a straight line measured by that measure. However this contradicts waveparticle duality since the smooth trajectory of a particle, a representation borrowed from classical physics, serves as an "absolute reference frame" to be depicted to it any quantum leap. Quantum measure is what can be used in one and the same way for both sides of duality and thus unifying them.

Quantum measure can serve to unify the set of probability distributions and that of numbers as well as the Schwartz distributions, or generalized functions, with the functions in a closer sense.

Quantum measure can be interpreted philosophically as well as that common measure of quantity and quality, which points out how "many" is per a unit of "much". If the unit of "much" is thought as a whole, quantum measurement shows how many of primary elements this whole contents.

Quantum measure can be obtained both from Lebesgue and Borel measure.

\section{Quantum invariance}

An initial way for quantum invariance to be defined is as follows: Any quantum coherent state before measurement cannot be a statistical ensemble excluding any "hidden variables" for the fundamental theorems of John von Neumann (1932, p. 167-173) and of Simon Kochen and Ernst Specker (1967, p. 70). Nevertheless the results after measurement constitute a statistical 
ensemble ordered in some finite values of a finite set of variables, e.g. the space-time coordinates of the measured results.

Consequently the following epistemological or ontological problems arise: How should map a coherent state (no statistical ensemble) before measurement with a statistical ensemble after measurement? Can just that mapping represent the original philosophical essence of quantum measurement? What are the definitive mathematical features of that mapping?

The answers have been long embarrassed by the following prejudices: The paradigm of measurement in classical physics supposes that the studied quantity has an exactly defined value before measurement, and the obtained statistical ensemble of the measured results is only due to various external disturbances or to the technical imperfections of the devices. The same model has been transferred implicitly and uncritically in quantum mechanics and measurements, and the following misleading philosophical interpretations have been made:

Quantum reality is incognizable or noncausal. It is created by or depends on its observer or measuring device and thus requires them. Quantum mechanics is incomplete and should complement by "hidden variables", yet unknown, to a future theory sharing cognizability, causality and independence from the observer and apparatus with the theories of classical physics and science. And so on. Their history is often called the philosophy of quantum mechanics. In fact all phenomena of entanglement studied by the theory of quantum information demonstrate bankruptcy of that philosophy of quantum mechanics since both "no hidden variables" and there are quantum correlations very well causally cognizable and independent from any observer or device.

The true pathway for the philosophy of quantum mechanics passes through the adequate generalization of 'measurement' to involve the discrepancy, due to the fundamental Planck constant, between any quantum coherent state and its statistical representation as a statistical ensemble by means of any measuring. That generalization neutralizing this discrepancy is meant as quantum invariance, which will be elucidated further.

The concept of measurement in classical physics may serve anyway as an initial reference frame, to which that generalization to be introduced. In fact the same problem of how the state before measurement to be mapped into the statistical ensemble of the measured results exists in classical physics and can be solved only for the above discrepancy is able to converge to zero: Indeed any statistical ensemble of results is finite and thus well-orderable while the corresponding physical quantity "before measurement" has to be smooth (and consequently continuous) so that its change to be described by differential equations. Only the axiom of choice is able to well-order it transforming it into the statistical ensemble of measured results. However the necessary collaboration of the axiom of choice is hidden since the difference between the real value before measurement and the corresponding element in the statistical ensemble of results converges to zero in principle for the Plank constant has no analog in classical physics.

That way out is closed to quantum mechanics: Any smooth quantity differs fundamentally from its measured value just for the Planck constant. However a usual "trick" in physics and mathematics is an arisen problem to be postulated as an axiomatic feature of the investigated system if it is consistent with the rest properties. So one has to require that any quantum coherent state is equivalent to the corresponding bounded, discrete, and consequently finite set of measured results, i.e. to a statistical ensemble, is an axiom in quantum mechanics.

A set-theory corollary is the curious invariance to the axiom of choice: Any coherent state excludes any well-ordering and thus excludes also the axiom of choice. However the above equivalence requires it to be equated to a well-ordered set after measurement and thus requires the axiom of choice for it to be able to be obtained. This is not a contradiction, though, since the 
statement (the axiom of choice) is in one situation (measurement), and its negation is in another (before measurement). Even the relation between the two situations does not generate contradictions, and it requires only the invariance in question.

In fact that invariance to the axiom of choice is well known in set theory as the so-called paradox of Skolem (1922). He introduces the relativity of 'set' meaning that any set of any power can be equivalently represented as a countable set utilizing the axiom of choice. Then if one considers a continuum in two axiomatics of set theory accordingly with or without the axiom of choice, the continuum would be invariant to the axiom of choice in the above sense. Furthermore Skolem states that the notions of finite or infinite set are thus relative (Skolem 1922, pp. 143-144) and in the present context this already reaches a set-theory interpretation of quantum invariance. Indeed if the limit of any series is given, the axiom of choice guarantees that its element which differs from the limit by some finite value has to exist necessarily and thus a continuum to be effectively replaced by a discrete set.

In turn that invariance implies fundamental conclusions in the philosophy of quantum mechanics as it can be generalized as the specific relation between reality and cognition in quantum mechanics and designated as invariance to choice. The invariance to choice would resolve a series of philosophical problems in a way, in which they vanish as their alternatives turn out to be invariant to each other and thus redundant. However the generalization of the particular viewpoint of the philosophy of quantum mechanics beyond its domain seems rather problematic.

As to the proper area it implies radical conclusions, too: The physical sense and meaning of information, of its quantity as well as the smooth transition between physics and the physical, on the one hand, and mathematics and the mathematical, on the other, can be easily deduced:

The quantity of information turns out to be that one for the invariance of choice and this can be demonstrated well by the unit of information, a bit. For example, a bit can be represented as an empty cell (as on the tape of a Turing machine), in which two disjunctive options such as either " 0 " or " 1 " can be written and read. Then the invariance of that maximally elementary choice is one bit: The empty sell as an original "coherent state" and both certain results (either written or read) are equated and thus invariant as one bit of information. The bit can be generalized from two to infinitely many disjunctive options, which can be written or read on an empty sell, and designated as a quantum bit or qubit. If those options are represented as the written or read values of a unit sphere (i.e. on the surface of a unit ball), Hilbert space, which is the fundamental mathematical structure of quantum mechanics, is portrayed as that tape of a quantum Turing machine with infinitely many qubits instead of the finite number of bits of a standard Turing machine. Furthermore any wave function is just one state of that quantum Turing machine and thus any physical process being quantum in general turns out to be also an exactly defined quantum computation and vice versa.

The corresponding generalization of information is quantum information, which can be thought as that quantity apt to measure the qualitative quantum invariance. Then one qubit can be defined as that unit of quantum invariance, which is able to equate a coherent state to a countable set of measurable values for the state. Entanglement can be defined as that case when a coherent state is equivalent to any true subset of that countable set, i.e. entanglement decreases the degrees of freedom so that some measurable values of the coherent state are forbidden due to the external influence of one or more other coherent states.

All phenomena of entanglement studied by the theory of quantum information are describable as the relation of a qubit (or qubits) to its subset (their subsets). The relation can be transformed into the ratio between the corresponding measures of the subset and set since a qubit is a continuum of disjunctive options with nonzero measure as the area of the unit sphere. 
So unlike the classically defined information, quantum information generates one derivative and additional metrics interpretable as the ratio between two qubits and thus as an angle between them and as an informational "curvature" available in all phenomena of entanglement.

The mathematical sense and meaning of that physically measurable ratio should be emphasized certainly: If the quantity of quantum information is visualized as a factor, which expresses a "much" as a "many" or "how many" per a unit of "much", that ratio being a finite number is the relation of an "infinitely many" to another "infinitely many" reducing the "common denominator" of one and the same "much". Thus quantum information allows of infinity to be represented physically and measured in an experimental way opening up to the horizon of an exact and quantitative science of infinity.

In particular gravity as it is mathematically represented in general relativity can be explained in terms of that ratio of qubits. These two qubits is enough to be accordingly the one of space-time position and the other of energy-momentum state in this position. Then their ratio will correspond to the space-time curvature in that point and to the value of gravitational field in it. Consequently gravity should have a quantum informational base and be relative to entanglement. Indeed entanglement and gravity can be seen correspondingly as the local and global aspect of one and the same, namely that variable metric relation embedded in quantum information.

That approach is consistent with the thermodynamic interpretation of general relativity (Jacobson1993) deducing it from the laws of classical thermodynamics and the Bekenstein bound (Bekenstein 1973). In fact the latter introduces mutual incommensurability of the quantities, which are conjugates according to quantum mechanics like energy and time since their differentials are inversely proportional rather than proportional as in classical mechanics. So general relativity can be considered both as a quantum thermodynamic theory and as a theory of quantum gravity. However both the former and the latter are paradoxically realized:

General relativity as a thermodynamic theory has an inverse statistical representation in the following sense: If statistical thermodynamics reduces the whole of a thermodynamic system and its quantities to some statistical ensemble of elements like atoms or molecules and the quantities of their mechanical motions, general relativity is forced to reduce the elements of its ensemble whichever it is to the state or states of the system. The true space-time can be accepted as a statistical ensemble relevant to that general relativity interpreted thermodynamically. This reminds of some return of the "ether" removed by special relativity as redundant but consistent with general relativity (Einstein 1920).

So the approaches of statistical thermodynamics and general relativity turn out to be complement to each other or in other words, both together constitute a joint approach addressing a cyclic structure, in which the states of the system are identified as its parts and vice versa transferring an isomorphism between the Gibbs and Boltzmann interpretation of statistical mechanics.

General relativity as a theory of quantum gravity would quantize gravity in an extremely unexpected way: It is forced to consider any quantum whole including the universe effectively as a single quantum and representing its internality in a continuous or even smooth way. Said otherwise, general relativity offers an alternative and complementary viewpoint to describe any quantum leap and thus any mechanical motion in that continuous or even smooth way. Furthermore, that viewpoint can be quantitatively described as another law of conservation, which is the complementary counterpart of the fundamental conservation of energy (mass) in the framework of a more general conservation of action consistent with general relativity, in which energy-momentum is conserved only locally. If conservation of energy is "per a unit of time", its 
counterpart expresses the conservation of time (time period of a wave), which would be "per a unit of energy" describing how the total energy of the system is distributed between its parts. Both conservations can be interpreted as revealing a side of wave-particle duality and quantitatively complementing to each other in conservation of action. If the common conservation of energy (mass or energy-momentum) offers the usual perspective of particles localized in space-time, the newly conservation of time understands the world as a collection of coherent waves or of interacting states of the universe.

If the qubit is interpreted philosophically as "how many a much is", the relation of two qubits as a ratio is anyway problematic since this supposes a reduction of the "much" in two different qubits. Allowing that reduction, one can obtain an exactly defined value of curvature in any space-time point as above, but rejecting it the result is the common wave-particle duality of quantum mechanics expressing itself in incommensurable conjugates as well. General relativity determines any quantity relative to that ratio of qubits as a "bound variable" while quantum mechanics remains it as an empty degree of freedom or as a "free variable". General relativity and quantum mechanics are on both sides of another unifying fence derivative from that of quantum invariance: The former allows of two relations of quantum invariance to be commensurable by identifying their "much" as joint, the latter does not. One can say that both express differently one and the same: Globally the ratio of any two conjugates has to remain a free variable while any location of it determines an exactly defined value "bounding" the free variable and decreasing the degrees of freedom by one as if a "hologram" is projected on the "screen" of space-time.

The same ratio though introduced by general relativity can be interpreted in terms of quantum mechanics if wave-particle duality is temporally suspended as the ratio of the unit of smooth motion and that of quantum leap. Then quantum mechanics can be seen as that generalization of general relativity, which is apt to comprise also the discrete mechanical motions together with all smooth ones involved by the principle of relativity (Einstein 1918). However in that case the curvature of space-time could be interpreted as an equivalent smooth mapping of the variable ratio of those units. Consequently general relativity includes implicitly also all quantum leaps in another way. What it cannot involve in principle is wave-particle duality since this would be removed its subject, gravity.

Thus the concept of quantum invariance allows of the relation between those two, most fundamental physical theories to be seen in a new way and furthermore, the problem of quantum gravity as well: General relativity and the Standard model underlain by quantum mechanics are on both sides of quantum invariance, which unifies them so. All three interactions comprised by the Standard model constitutes a finite set of groups (i.e. symmetries) having representations in the group of the linear automorphisms of Hilbert space. Pseudo-Riemannian space utilized by general relativity to describe gravity can be obtained from Hilbert space by three reversible operations hinting the equivalence of both spaces under certain conditions: The first one transforms the actually infinitely dimensional Hilbert space into a process. The second replaces the independent variable of energy (frequency) with its reciprocal, time. The third one deforms both spheres and their relative position to each other corresponding to any given moment of time. If one omits to reverse the third operation, but does reverse the first and second one Hilbert space will be transformed into Banach space, and it will be able to represent entanglement as a mathematical formalism thus clearing up the close link between the structures associated with gravity and entanglement accordingly.

The concept of quantum invariance underlies that of quantum information and allows of revealing its philosophical and physical meaning as the relation of an unordered quantum "much" 
(i.e. a coherent state) and a well-ordered "many" of the measured results (i.e. a statistical ensemble). So quantum invariance clears up quantum information as a relation of ordering and opens up to a new horizon, in which all physical processes and phenomena can be interpreted as quantum computations realizing relevant operations and algorithms on quantum information. So one can peer into the "black box" of the physical world and discern its hidden mechanism, which turns out to be informational. Furthermore that concept shows up how quantum information is able to unify all known physical interactions situating gravity, on the one hand, and weak, strong and electromagnetic interaction, on the other hand, on both sides of quantum invariance. It needs entanglement to accomplish that unification and thus legitimates it as the bridge between these two sides and perhaps as a new fundamental interaction. Consequently the notion of quantum invariance has fundamental meaning for the epistemology of quantum mechanics and information.

\section{The Nonlocality of Quantum Information}

All phenomena of entanglement demonstrate that quantum information: (1) cannot be localized in space-time; (2) can make any item localized in space-time to interact with others even beyond of the light cone; (3) restricts the degrees of freedom of the interacting items as a classical force or field, but unlike them probabilistically in space-time. All this enables quantum information to be considered as a unifying generalization both of information and of space-time localizable particles and fields and thus of computational and physical processes as well as both of information and its carrier.

The notion of quantum information allows of quantum mechanics to be reinterpreted as a measure theory involving a corresponding measure and qubits as its units. That measure unifies continuous and discrete quantities and thus conditions the nonlocality of all measured by it. Quantum information can be the universal physical substance of the world, which underlies the four interactions.

The nonlocality of quantum information will be considered in a narrow and in a broad sense:

In the former it refers to all space-time violating Lorentz invariance and restricting the degrees of freedom (DF) of some quantum systems anywhere they are.

In the latter it generalizes the invariance of classical information and computation to space-time localization or to their carriers, which are always localized in space-time.

Quantum measure underlies and conditions the nonlocality of quantum information allowing of all quantum mechanics to be reinterpreted in its terms. In turn the adoption of quantum measure is needed by wave-particle duality and by the requirement of a common measure both of discrete (quantum) and continuous (smooth) quantities. In final analysis it leads to quantum information to be able to be accepted as the universal substance of the physical world in a philosophical sense.

The theory of quantum information arose historically as part of quantum mechanics studying the phenomena of entanglement. Two famous papers (Einstein, Podolsky, Rosen 1935; Schrödinger 1935) were the beginning. The former is well known as "The EPR paradox", and the latter as "The Scrödinger's cat paper". Both deduce that the mathematical formalism of quantum mechanics requires a special kind of interaction between two or more quantum systems, which seems as if "spooky" or revitalizing "action at a distance". Albert Einstein, Boris Podolsky, and Nathan Rosen showed its extraordinary properties as a proof of the alleged "incompleteness of quantum mechanics", and Erwin Schrödinger called them "verschränkten zustände" (entangled states). 
The classical paper of John Bell (1964) revealed that if those states exist in nature, they imply experimentally observable effects. Entanglement was experimentally confirmed by John Clauser and Michael Horne (1974), Allain Aspect, Philippe Grangier, and Gérard Roger (1981; 1982), and by many others afterwards. All this leaded to the theory of quantum information to appear within quantum mechanics to study all phenomena of entanglement.

The theory of quantum information can be differentiated as the quantum physics of information or as the quantum part of the physics of information. However it allows of quantum mechanics to be newly interpreted as an informational theory about a kind of generalized information, which is quantum. Quantum information and thus information underlies the world according to that interpretation since quantum mechanics underlies the world physically.

Quantum information will be defined as a theory of information in Hilbert space shared with quantum mechanics and thus transferring immediately physical and informational processes and phenomena to each other even "one-to-one".

Hilbert space can be considered as the simplest generalization and thus unification both of natural numbers and Euclidean space traditionally studied by arithmetic and geometry accordingly. Both investigate quantities, but they are correspondingly discrete in the former, and continuous in the latter. Their measures are quite different: the number of units, on the one hand, and lengths, areas, volumes, on the other hand.

Quantum information being information in Hilbert space can be thought as the unification apt to measure jointly the quantity of information both in discrete and in continuous quantities adopting a relevant measure unit such as a qubit (i.e. quantum bit)

\subsection{The nonlocality of quantum information in a narrow sense}

\subsubsection{Quantum information and entanglement}

Entanglement is commonly defined as kind of interrelation of three or more Hilbert spaces usually interpreted in quantum mechanics as corresponding to a quantum system and two or more its subsystems. Entanglement means that no Hilbert space of those in question can be factorized to a tensor product of the rest. The same interpreted as to quantum mechanics sounds so:

The Hilbert space associated with a quantum system cannot be factorized to the Hilbert spaces associated with two or more given subsystems of it. Those subsystems are designated as entangled. As a result their wave functions are deformed sharing and restricting mutually DF, i.e. possible states of at least two of the subsystems. Quantum correlations violating the Bell (1964) inequalities and other similar ones discovered later appear between entangled subsystems. Quantum correlations can exceed the maximal rate possible for any classical correlations and thus that excess is a sufficient (but not necessary) condition of quantum correlations allowing them to be experimentally verified.

Entanglement and quantum correlations are due to the unification of two quite heterogeneous kinds of measure in Hilbert space enabling to be jointly measured discrete and continuous quantities.

One needs to reformulate Hilbert space in terms of the units of quantum information, i.e. qubits, to demonstrate the quantum information as information in Hilbert space and then entanglement as a specific quantum-informational interaction.

The definition of a qubit is:

1 qubit $=\alpha|0\rangle+\beta|1\rangle$, where $\alpha, \beta$ are complex numbers such that: $|\alpha|^{2}+|\beta|^{2}=1$, and $|0\rangle,|1\rangle$ are two orthogonal spaces or their orthonormal bases, e.g. any two consecutive "axes", $\mathrm{n}^{\text {th }}$ and $\mathrm{n}+1^{\text {th }}$ one of Hilbert space such as $e^{i n \omega}, e^{i(n+1) \omega}$. 
Given any vector $\vec{V}$ of the complex Hilbert space with components $C_{1}, C_{2}, \ldots, C_{n}, C_{n+1}, \ldots$ in "axes", $1^{\text {st }}, 2^{\text {nd }}, \ldots, \mathrm{n}^{\text {th }}, \mathrm{n}+1^{\text {th }}, \ldots$, one can construct a one-to-one mapping of it into its representation in qubits, e.g. so:

If both consecutive components, $C_{n}=0$ and $C_{n}=0: \alpha_{n}=0, \beta_{n}=0$ (conventionally).

If the consecutive components, $C_{n} \neq 0$ or $C_{n+1} \neq 0$ :

$$
\alpha_{n}=\frac{C_{n}}{\sqrt{\left|C_{n}\right|^{2}+\left|C_{n+1}\right|^{2}}}, \beta_{n}=\frac{C_{n+1}}{\sqrt{\left|C_{n}\right|^{2}+\left|C_{n+1}\right|^{2}}} .
$$

Furthermore any qubit is isomorphic to a usual, three-dimensional unit ball and its value to a pair of points: Both of them can be ones of two orthogonal great circles, or the one of the ball, the other of the unit sphere (i.e. the surface of the ball). However the power of the set of all points on the unit sphere is the same as that of those pairs of points as above, and a qubit can be equivalently visualized as a choice of a point of the unit sphere, i.e. a choice of an element of a continuum, i.e. an uncountable set.

All this enables a set-theoretical representation of a qubit: as any choice (as its value) of an element of any uncountable set. This involves necessarily the axiom of choice, after which any uncountable set can be equivalently considered as a countable set, which can be infinite or even finite (Skolem 1922). However an immediate corollary of the theorem of Kochen and Specker (1968) excludes any representation of a qubit into a bit or into any finite series of bits. Thus the only opportunity to be reconciled the Kochen - Specker theorem and Skolem's "paradox" implied by the axiom of choice is that: The qubit is considered as a choice among an enumerable number of disjunctive alternatives, which is equivalent to any power of an infinite set of alternatives just because of Skolem's "paradox".

Furthermore entanglement can be interpreted properly set-theoretically as a specific property involved for the intersection of two or more infinite sets of alternatives.

Entanglement can be also visualized as an arbitrary non-empty intersection of two unit balls (or spheres): If two qubits are entangled they have to share and thus to restrict each other for any value belonging to the intersection. However the two or more entangled qubits do not have any space-time localization: Entanglement is nonlocal.

\subsubsection{Quantum information and space-time}

Another question arises then: How should one map the nonlocal quantum information onto space-time, which is a continuous (even smooth) medium and thus it is locally defined? After quantum information is defined as information in Hilbert space, the question can be simplified as the relevant mapping of Hilbert space onto Minkowski space invariant to the quantity of quantum information:

Following the qubit representation of Hilbert space and defining the measure unit of quantum information as the basis of Hilbert space, the quantity of quantum information should coincide with a point in it, which can be interpreted as a wave function, i.e. as a state of a quantum system.

Thus the nonlocality of quantum information is the same as that of wave function: Any point of space-time has a probability for a quantum item with a given wave function to be localized in it according to Born's interpretation. Here however "any point of space-time" can mislead: One should mean any point of space-time in any time moment, i.e. any point of Newton's absolute space in fact. This says that any point of space-time has to include somehow all space-time within it. The nonlocality of quantum information or that of wave function 
expresses just this. The probabilistic interpretation of wave function reconciles that nonlocality with the exact local definiteness of any point of space-time.

\subsubsection{Quantum information and Lorentz invariance}

At first glance the nonlocality of quantum information should violate Lorentz invariance as points beyond the light cone can influence to those within it by entanglement. Indeed the Schrödinger equation is not Lorentz invariant though it does not excludes it as the Dirac equation displays. Einstein objected to quantum mechanics also for the "spooky" action at a distance as he, Podolsky and Rosen demonstrated quite clearly in their joint paper. However there is another viewpoint:

One can obtain the Lorentz invariant Minkowski space measuring Newton's absolute space by means of quantum measure expanding it in time by the speed of light in vacuum. Then one can see Hilbert space "expanding" in frequency and Minkowski space as dual counterparts both delivered by measuring Euclidean space by quantum measure only differently interpreting Euclidean space physically: as momentum space for Hilbert space or as position space for Minkowski space. The quantity of quantum information will seem as a wave function in the former or as a world line in the latter. Thus any wave function corresponds one-to-one to a world line by the formal transformation between time and frequency, i.e. by that between two corresponding points of Hilbert space and its dual one, which are identical mathematically but with different interpretations, which are even complementary in the sense of quantum mechanics.

Consequently Lorentz invariance is almost automatically conserved in quantum information for Hilbert space is equivalent to Minkowski space as above. In that sense the nonlocality of Hilbert space originates from its simultaneous consideration unlike Minkowski space usually interpreted locally in time, moment by moment.

However if one speaks of the violation of Lorentz invariance, he or she means some phenomena of entanglement rather than the quantum information itself. Indeed entanglement can be visualized as any deformation of Hilbert space as to the joint system unlike those of the entangled subsystems compounding it. That deformation can be transformed into the corresponding deformation of Minkowski space changing it into pseudo-Riemannian space thus violating Lorentz invariance also locally unlike Minkowski space, to which Lorentz invariance is not valid in a global sense as above:

Indeed any qubit or the mutual orthogonality of any qubits can be deformed violating Lorentz invariance. All that can be equivalently reflected by some corresponding deformation of the wave function.

\subsubsection{Quantum information and physical interaction}

Entanglement can be expressed as a kind of interaction sharing and generalizing a few properties of the usual physical interaction. The latter changes certain physical quantities, and the former restricts DF of that physical system influenced. So one should clear up how the notion of restricted DF can generalize that of physical quantity as it is utilized in quantum mechanics and designated as 'observable'. Its definition is: $\bar{A}=\int_{-\infty}^{+\infty} \Psi(x) \hat{A}\left[\Psi^{*}(x)\right] d x$, where $\bar{A}$ is the quantity, $\hat{A}$ is the self-adjoint operator in Hilbert space corresponding to the quantity, $\Psi(x)$ is the wave function of the system, and $\Psi^{*}$ is its conjugate, $x$ designates the generalized coordinates.

What happens to this quantity if the system characterized by it undergoes entanglement? It can be expressed in a few equivalent ways in the above formula, e.g. as any transformation of $\Psi(x) \rightarrow \Psi_{t}(x)$ or $\Psi^{*}(x) \rightarrow \Psi_{t}^{*}(x)$ such that $\left|\Psi_{t}(x) . \Psi_{t}^{*}(x)\right|<1$ or as any non-identical transformation $\hat{A} \rightarrow \hat{A}_{t}$. The change $\bar{A} \rightarrow \bar{A}_{t}$ is a sufficient condition of entanglement, which can be designated as that entanglement, which is observable by this quantity. What follows to the end 
of the paragraph is restricted only to that "observable entanglement". It would lead to some finite increase $\Delta \bar{A}=\overline{A_{t}}-\bar{A}$ of the quantity interpretable as some nonzero external physical interaction. The converse is also true: given any finite change of any physical quantity of any system, it can be interpreted both as some physical interaction and as some entanglement of the system with its environment. Consequently entanglement and thus quantum information underlies any physical interaction explaining the most general mechanism of how it can work.

\subsection{The nonlocality of quantum information in a broad sense}

This means the way for quantum information being nonlocal in a narrow sense as above to refer to relevant entities, which unlike it are well localizable in space-time, such as physical existence, physical processes or the carrier of information

While classical information obeys the physical at all, i.e. the physical existence, process, carrier and their localizability in space-time, all of them obey quantum information thus unifying them in the following sense:

Any wave function is a value of quantum information measured by qubits as one can be convinced above. Furthermore the identity of physical existence and any wave function seems obvious: Any existing is a quantum system and the state of which is described by a wave function.

Quantum computation can be considered as the computation in Hilbert space and represented by a quantum Turing machine, in which bits are replaced by qubits though those a few operations on a single qubit, i.e. a single cell of a quantum Turing tape can be analogical to those of a standard Turing machine implemented on a single bit, i.e. on a single cell of it. They are (the few differences are italicized):

"Write a value of a qubit!" for "Write a value of a bit!"

"Read a value of a qubit!" for "Read a value of a bit!"

"Go to the next cell!" for the same.

"Stop!" for the same.

The reversibility of quantum computation is hidden in any single qubit in that model of a quantum Turing machine after the "write/ read commands" require a choice among all points of a continuum (an uncountable set) and thus the axiom of choice. Indeed the input/ output of data into a quantum system are irreversible though all its states between them are thoroughly reversible.

Conclusions:

Cognition arranges reality as our knowledge of it. Epistemology is what studies that arrangement. Quantum mechanics manages to generalize these common facts in a way contradicting the prejudices of common sense. It shows the following:

1. Quantum measurement and thus cognition is a mapping of all states inorderable in principle, before measurement, and the well-ordered sets of results constituting statistical ensembles, after measurement.

2. The axiom of choice is necessary to be involved to guarantee that mapping in any case. Nevertheless the states before measurement exclude involving the axiom of choice as to them. Consequently the axiom of choice should not be involved in the description before measurement, but should be involved in that after measurement. Both descriptions should share one and the same mathematical formalism.

3. Hilbert space manages to satisfy those requirements seeming impossible simultaneously. One can coin the metaphor of the identical "hypostases" of Hilbert space, each one of which is "embodied" in one of the two identical dual Hilbert space: The one "hypostasis" 
is unorderable and it is before measurement, and the other being well-ordered after all is after measurement. This do not generate any contradiction, though: For both dual spaces cannot be given simultaneously, or they are "complementary" if one utilize the term of their interpretation in quantum mechanics.

4. In fact the quantity of information involves the relation between an "empty state" (or empty cell" as well as "free variable") and the number of all different possible states (or "writable values" as well as "bound variable"), in which the empty state (cell, free variable) can turn out to be. Consequently the notion of quantum information is a natural generalization in the case where the number of different possible states is infinite, and its unit generalizes that of a bit for classical information so that a quantum bit (qubit) is a choice among the infinitely many equiprobable alternatives of choice.

5. Thus the notion of quantum information describes well the relation between the two "hypostases" of Hilbert space and furthermore, quantum measurement and cognition. It eliminates the alleged and wrong subjectivity of quantum reality ostensibly needing an observer, to be or to exist.

6. Quantum information suggests a new and more general viewpoint to quantum mechanics, which can be conventionally designated as covariant since it interprets quantum mechanics as a theory of a new kind of measure apt to measure discrete and continuous sets together in shared relevant units, qubits.

7. The same approach of quantum information allows to be unified space-time and quantum nonlocality as the "hypostases" of Hilbert space and to be quantitatively measured together. This demonstrates the common structure of quantum nonlocality and cognition. 


\section{References:}

Aspect, Alain, Philippe Grangier, and Gérard Roger (1981). "Experimental tests of realistic local theories via Bell's theorem". In: Physical Review Letters 47.7, pp. 460-463.

(http://people.isy.liu.se/en/icg/jalar/kurser/QF/assignments/Aspect1981.pdf)

Aspect, Alain, Philippe Grangier, and Gérard Roger (1982). "Experimental Realization of Einstein-Podolsky-Rosen-Bohm Gedanken Experiment: A New Violation of Bell's Inequalities". In: Physical Review Letters 49.2, pp. 91-94.

(http://qudev.ethz.ch/content/courses/phys4/studentspresentations/epr/aspect.pdf)

Bekenstein, Jacob (1973). "Black Holes and Entropy". In: Physical Review D 7.8, pp. 23332346.

(http://prd.aps.org/abstract/PRD/v7/i8/p2333 1)

Bell, John (1964). "On the Einstein - Podolsky - Rosen paradox". In: Physics (New York) 1.3, pp. 195-200.

(http://philoscience.unibe.ch/documents/TexteHS10/bell1964epr.pdf)

Clauser, John and Michael Horne (1974). "Experimental consequences of objective local theories". In: Physical Review D 10.2, pp. 526-535.

(http://prd.aps.org/abstract/PRD/v10/i2/p526 1)

Eninstein, Albert. (1918). "Prinziplelles zur allgemeinen Relativitätstheorie". In: Annalen der Physik 55.4, pp. 241-244.

Einstein, Albert (1920). Äther und Relativitätstheorie. Berlin: Springer.

(http://www.mahag.com/rede.htm )

Einstein, Albert, Boris Podolsky, and Nathan Rosen (1935). "Can Quantum-Mechanical Description of Physical Reality Be Considered Complete?" In: Physical Review 47.10, pp. 777780.

(http://www-f1.ijs.si/ ramsak/teaching/eprbohr.pdf)

Jacobson, Ted (1995). "Thermodynamics of Spacetime: The Einstein Equation of State". In: Physical Review Letters 75.7, pp.1260-1263.

(http://arxiv.org/pdf/gr-qc/9504004.pdf)

Kochen, Simon, and Ernst Specker (1968). "The problem of hidden variables in quantum mechanics". In: Journal of Mathematics and Mechanics 17.1, pp.59-87.

(http://www.iumj.indiana.edu/IUMJ/FTDLOAD/1968/17/17004/pdf)

Neumann, John von (1932). Mathematische Grundlagen der Quantenmechanik. Berlin: Springer. Schrödinger, Erwin (1935). "Die gegenwärtige situation in der Quantenmechanik". In: Die Naturwissenschaften 23.48, pp. 807-812; 23.49, pp. 823-828; 23.50, pp. 844-849.

(http://wwwthep.physik.uni-mainz.de/ matschul/rot/schroedinger.pdf)

Skolem, Thoralf (1922). "Einige Bemerkungen zur axiomatischen Begründung der Mengenlehre". In: Selected works in logic of Thoralf Skolem (ed. E. Fenstad), Oslo: Univforlaget (1970), pp. 137-152. 